\title{
Strategy-Controlled \\ Reduction and Narrowing
}

\author{
Peter Padawitz \\ Fakulut fur Mathematik und informatik \\ Universität Passau
}

\begin{abstract}
The inference rules "reduction" and "narrowing" are generalized from terms resp. equations to arbitrary atomic formulas. Both rules are parameterized by strategies to control the selection of redices. Church-Rosser properties of the underlying Horn clause specification are shown to ensure both completeness and strategy independence of reduction. "Uniformity" turns out as the crucial property of those reduction strategies which serve as complete narrowing strategies. A characterization of uniformity (and hence completeness) of leftmost-outermost narrowing is presented.
\end{abstract}

\section{INTRODUCTION}

Term reduction (or rewriting) is the process of iterated subterm replacement according to a set of "oriented" equational axioms (cf. / Huet, oppen/). Narrowing is a method to solve an equation $t \equiv t^{\prime}$ by transforming $t \equiv t^{\prime}$ into an equation $u \equiv u^{\prime}$ such that $u$ and $u$ are unifiable terms. Each transformation step substitutes into terms to obtain lefthand sides of axiom instances, which are then replaced by the corresponding righthand side instances. A solution of $t \equiv t$ is given by the composition over the resulting sequence of substitutions (cf. e.g. /Slagle/, /Lankford/, /Fay/, /Hullot/). Reduction and narrowing can be generalized in several respects:

- Starting from a Horn clause specification (with identity) we describe reduction and narrowing as transformation rules for goals, i.e. for sets of atomic formulas (where, of course, equations are a special case).

- As a consequence, narrowing can be regarded exactly as reduction plus substitution, and therefore strategies to control redex selection can first be associated with reduction and later be lifted to narrowing.

- Goal reduction allows us to consider different notions of "reducedness", which correspond to different Church-Rosser properties.

- Goal narrowing generalizes "conditional narrowing" (cf. /HuBmann/, /Fribourg/).

Aspects leading to and resulting from this approach are discussed in detail in /Padawitz/. The paper at hand focuses on goal reduction and narrowing with regard to strategies that control the selection of reduction resp. narrowing 
redices. While reduction redices are instances of lefthand sides of (conditional) equations, narrowing redices are prefixes of possible reduction redices.

For instance, let AX consist of the (conditional) equations $c \equiv$ true and (*)

$$
\tau(s(y)) \equiv \tau(y) \Leftarrow y \neq 0
$$

The goal $\gamma=\{\tau(s(s(0))) \cup c \equiv$ true $\}$ has two AX-reduction redices, namely $c$ and $\tau(s(s(0)))$. Replacing them by the righthand side instances of $c \equiv$ true resp. (*) leads to the goais

$$
\delta=\{\tau(s(s(0))) \vee \text { true } \equiv \text { true }\} \quad \text { resp. } \varphi=\{\tau(s(0)) \vee c \equiv \text { true, } s(0) \neq 0\} .
$$

Let $\gamma^{\prime}=\{\tau(s(0)) \vee c \equiv$ true $\}$. The only reduction redex of $\gamma^{\prime}$ is c because for $\tau(s(0))$ to be a reduction redex the corresponding premise instance of (*), namely $0 \neq 0$, is not $A x$-reducible. However, there is a prefix of $\gamma^{\prime}$, namely

$$
\gamma^{\prime \prime}=\{\tau(s(z)) \vee c \equiv \text { true }\}
$$

with narrowing redex $\tau(s(z))$. Consequently, leftmost-outermost narrowing would select the redex $\tau(s(z))$ of $\gamma^{\prime \prime}$, while leftmost-outermost reduction would choose the redex $c$ of $\gamma^{\prime}$. Hence - with respect to AX - leftmostoutermost narrowing is not uniform with leftmost-outermost reduction.

The paper is organized as follows: Section 2.1 presents syntactical preliminaries. Section 2.2 provides the semantical background: the Horn clause calculus, the deductive theory and conservative extensions, which result from splitting specifications into base (or "constructor") parts and extension parts. Conservative extensions allow us to restrict reductions to applications of extension axioms (cf. section 3.1). Section 3.2 develops the proof of reduction strategy independence in the presence of a Church-Rosser specification (Theorem 3.2.2). Section 3.3 is concerned with narrowing and aims at Theorem 3.3.1, which says that narrowing controlled by uniform strategies is complete - provided that we work with Church-Rosser specifications. Chapter 4 discusses the consequences of uniformity, presents a sufficient condition on specifications to make leftmost-outermost narrowing uniform (Lemma 4.2), provides a way to extend specifications such that this condition holds true (Lemma 4.4) and closes with a completeness result for leftmost-outermost narrowing (Theorem 4.5 ).

\section{HORN CLAUSE THEORIES}

\subsection{Syntax}

A signature $S \mid G=\langle S, O P, P R\rangle$ consists of a set $S$ of sorts and two $S^{+}$-sorted sets $O P$ and PR the elements of which are called operation and predicate symbols, respectively. 5-sorted operation symbols are called constants. For $\sigma$ $\epsilon O P_{w s}$ with $w \in S^{*}$ and $s \in S, w$ is called the arity and $s$ the coarity of $\sigma$. 
Typical operation symbols are $\sigma$ and $\tau$, typical predicate symbols are $P$ and $Q$.

General Assumption we suppose that a signature $S \mid G=\langle S, O P, P R\rangle$ is given. For all $s \in S$, $P R_{S s}$ implicitly contains a predicate symbol $\equiv_{s}$ called the equality predicate for $s$. Furthermore, we fix an $s$-sorted set $X$ of variables such that for all $s$ in $S, X_{s}$ is countably infinite.

The S-sorted set I(SIG) of terms over SIG is recursively def ined as usual:

- For all $s \in S_{s} O P_{s} \cup X_{5} \subseteq T(S G)_{s}$, i.e. constants and variables are terms,

- for all $w \leqslant S^{*}, s \in S, \sigma \in O P_{w s}$ and $t \in T(S I G)_{W}$, ot $\in T(S I G)_{s}$.

Typical variables or sequences of variables are $x, y$ and $z$. Typical terms or sequences of terms are $t, u$ and $v$. root $(t)$ denotes the leftmost symbol of $t$. The sort of $t$ coincides with the coarity of root( $t)$

The partial function label: $T(S I G) \times \mathbb{N}^{*} \rightarrow$ OPuX is defined by

- label $(t, \varepsilon)=\operatorname{root}(t)$

- label $\left(\sigma\left\langle t_{1}, \ldots, t_{n}\right\rangle, i w\right)=$ label $\left(t_{i}, w\right)$ for all $1 \leq i \leq n$.

If label $(t, w)$ is defined, say label $(t, w)=\sigma$, then $w$ is an occurrence of $\sigma$ in $t$. $\operatorname{var}(t)$ denotes the set of variables occurring in $t . t$ is ground if $\operatorname{var}(t)$ is empty. The set of ground terms over SIG is denoted by GT(SIG).

General Assumption (continued) For ali $s \in S, G T(S \mid G)_{S}$ is nonempty.

Let $A$ be an 5 -sorted set. The set of S-sorted functions from $X$ to $A$ is denoted by $A^{x} . f \leqslant T(S G)^{x}$ is called a substitution over SIG. $f$ is of ten written as the set of all expressions $t / x$ - read as " $t$ for $x "$ - such that $f(x)=t \neq x$. f $\epsilon$ $G T(S I G) x$ is called a ground substitution over SIG. Typical substitutions are $f, g$ and $h$. Some readers may not be familiar with the functional view on substitutions. Here the names "f", "g", etc. do not stand for operation symbols. The reason for our choice of notation is to clearly separate functions from symbols and to adopt the traditional notation for functions in mathematics, while keeping Greek letters for names of syntactical objects like operation symbols and formulas.

Let $t$ be a term and $g$ be a substitution. Then $t g]$ the instance of $t$ by $g$, is the term resp. substitution obtained from t by simultaneously replacing each occurrence of a variable $x$ in $t$ by $g(x)$. Vice versa, $t$ is called a prefix of $t[g]$. Consequently, for a substitution $f, f[g]$ is the substitution defined by $f[g](x)=$ $f(x)[g]$ for all $x \in X$, and $f$ is a prefix of $f[g]$. Given terms $t$ and $t^{\prime}, f$ unifies $\left\langle t, t^{\prime}\right\rangle$ or $f$ is a unifier of $\left\langle t, t^{\prime}\right\rangle$ if $t[f]=t^{\prime}[g]$ for some substitution $g$. A unifier $f$ of $\left\langle t, t^{\prime}\right\rangle$ is most general if $f$ is a prefix of every unifier of $\langle t, t\rangle$. The compositiongof of two substitutions $f$ and $g$ is defined as usual by $g \circ f=f[g]$.

Let $w \in S^{+}, P \in P R_{w}$ and $t \in T(S I G)_{w}$. $P t$ is called an atom over SIG. If $P$ is an 
equality predicate, say $\equiv_{5}$, and $t=\left\langle t_{0}, t_{1}\right\rangle$, then Pt is an equation over SIG, written as $t_{0} \equiv_{5} t_{1}$ or $t_{0} \equiv t_{1}$. The set of atoms over SIG are denoted by At(SIG). A goal over SIG is a subset of At(SIG). Goal(SIG) stands for the set of goals over SIG. Typical atoms are $p$ and $q$. Typical goals are $\gamma, \delta$ and $\lambda$. The function label is extended to atoms $p=p\left\langle t_{1}, \ldots, t_{1}\right\rangle$ and goals $\gamma$ as follows:

- label $\left(P\left\langle t_{1}, \ldots, t_{p}\right\rangle, w\right)=u\left\{\right.$ label $\left.\left(t_{i}, w\right) \mid 1 \leq i \leq n\right\}$,

- label $(\gamma, w)=u\{$ label $(p, w) \mid p \in \gamma)$.

$\operatorname{var}(p)$ and $\operatorname{var}(\gamma)$ denote the set of variables occurring in $p$ resp. $\gamma$. p resp. $\gamma$ is ground if $\operatorname{var}(p)$ resp. $\operatorname{var}(\gamma)$ is empty. instances, prefixes and unifiers of atoms and goals are defined analogously to instances, prefixes resp. unifiers of terms.

Note the difference between an equation $t \equiv t$ ' and the syntactical equality of $t$ and $t$ ", which is expressed by the "meta-equation" $t=t$. The notation used here follows the same principle as our notation for substitutions (see above): When reasoning about syntactical entities we prefer symbols which are used in mathematics, while the syntactical entities themselves are denoted by other symbols. Unfortunately, many authors do just the other way round and, e.g., use $\equiv$ for the syntactical equality of terms.

The type of formulas we adopt to axiomatize data types are definite (or positive) Horn clauses, briefly called clauses: A clause over SIG consists of an atom $p$ and a goal $\gamma$ over SIG, written as $p \Leftarrow \gamma$ or $p \Leftarrow p_{1}, \ldots, p_{n} . p$ and $\gamma$ are the conclusion and the premise of $p \Leftarrow \gamma$, respectively. If $\gamma$ is empty, we omit the implication sign $\Leftarrow p \Leftarrow \gamma$ is ground if $p$ and $\gamma$ are ground. If $p$ is an equation, then $p \Leftarrow \gamma$ is a called a conditional equation. (By previous definitions, a clause with empty premise is an atom and a conditional equation with empty premise is an equation.) Let $A X$ be a set of clauses over SIG. The pair $\langle S I G, A X\rangle$ is called a specification.

\subsection{The Horn clause calculus}

General Assumption (continued) We suppose that a signature $S I G=\langle S, O P, P R\rangle$ and a specification $\langle S \mid G, A X\rangle$ are given.

A slG-structure A consists of

- an S-sorted carrier set denoted by A,

- an element $\sigma^{A} \in A_{5}$ for each constant $\sigma \in O P_{5}$,

- a function $\sigma^{A}: A_{W} \rightarrow A_{5}$ for each $w \in S^{+}, s \in S$ and $\sigma \in O P_{w s}$,

- a relation $P^{A} \subseteq A_{w}$ for each $w \in S^{+}$and $P \in P R_{w}$.

If for all $s \in S$, \# Is $_{S}$ is the equality on $A_{5}$, then $A$ is a SlG-structure with identity. The 5-sorted evaluation mapping for $A$, eval $^{A}: G T(S I G) \rightarrow A$, is recursively defined as follows: 
- For all $s \in S$ and $\sigma \in O P_{s}$, evale $\left.\right|_{5} ^{A}(\sigma)=\sigma^{A}$,

- for all $w \in S^{+}, s \in S, \sigma \in O P_{w s}$ and $t \in G T(S 1 G)_{w}$. evale $(\sigma t)=\sigma^{\text {fooval }}(t)$.

If eval ${ }^{A}$ is surjective, then $A$ is term-generated. Let $S G(X)=\langle S, O P \cup X, P R\rangle$ and $b$ $\in A^{x}$. A becomes a $S I G(X)$-structure $A(b)$ by defining $X^{A(b)}=b(x)$ for all $x \in X$. Vice versa, $b$ is extended to an $S$-sorted function $b^{*}: T(S I G) \rightarrow A$ by $b^{*}(t)=$ eval ${ }^{A(b)}(t)$. Given a goal $\gamma, b$ is a solution of $\gamma$ in $A$ if for all atoms $P t \in \gamma, b^{*} t$ $\epsilon P^{A}$. A satisfies $A X$ or $A$ is a SIG-model of $A X$ if for all clauses $p \Leftarrow \gamma$ in $A X$, the solutions of $\gamma$ in A are solutions of $p$ in A. A class $C$ of SIG-structures satisfies $A X$ if all $A \in C$ satisfy $A X$. Mod(SIG, $A X)$ denotes the class of all S1G-models of AX with identity.

Let $C$ be a set of clauses and $c$ be a single clause. The Horn clause calculus consists of the following derivation rules where " $\mathrm{C} t \mathrm{C}$ " stands for " $\mathrm{C}$ is derivable from $\mathrm{C}^{\prime \prime}$.

Substitution Rule for clauses $c$ and substitutions $f,\{c\} \vdash c[f]$.

Cut Rule For atoms $p, q$ and goals $\gamma, \delta,\{p \Leftarrow \gamma \cup\{q\}, q \Leftarrow \delta\} \vdash p \Leftarrow \gamma \cup \delta$.

Composition Rule $\mathrm{C} \vdash \mathrm{c}$ and $\mathrm{C}^{\prime} u\{c\} \vdash \mathrm{c}^{\prime}$ imply $\mathrm{CuC} \vdash \mathrm{c}^{i}$.

The set EAX of equality axioms consists of all clauses of the form

$$
\begin{aligned}
& x \equiv x \\
& y \equiv x \Leftarrow x \equiv y \\
& x \equiv z \Leftarrow x \equiv y, y \equiv z \\
& o\left\langle x_{1}, \ldots, x_{n}\right\rangle \equiv \sigma\left\langle y_{1}, \ldots, y_{n}\right\rangle \Leftarrow x_{1} \equiv y_{1}, \ldots, x_{n} \equiv y_{n} \\
& P\left\langle y_{1}, \ldots, y_{n}\right\rangle \Leftarrow P\left\langle x_{1}, \ldots, x_{n}\right\rangle, x_{1} \equiv y_{1}, \ldots, x_{n} \equiv y_{n} .
\end{aligned}
$$

The deductive theory of $\langle S \mid G, A X\rangle$, DTh $(S|G, A X\rangle$, is given by all goals over SIG, which are derivable from AXUEAX using the Horn clause calculus. Goals in the deductive theory of $\langle S \mid G, A X\rangle$ are briefly called $\langle S| G$, AX $\rangle$-theorems.

If all predicate symbols of $S I G$ are equality predicates, then DTh(SIG, AX) agrees with the congruence relation on $T(S I G$ ) generated by $A X$ (cf. /Ehrig, Mahr/, pp. $77 \mathrm{f}$. and 119, or /Kaplan/, Thm. 2.3). In general, for all goals $\gamma, \gamma$ is a $\langle S G, A X\rangle$-theorem iff Mod(SIG, AX) satisfies $\gamma$ (cf. / Padawitz/, Thm. 4.2).

Reduction and narrowing will be regarded as refinements of the Horn clause calculus. However, completeness of these refinements depends on certain requirements to the specification based on the suitable splitting into a base specification and an extension. The base specification contains all sorts, "constructor" operations and all predicates of $\langle S \mid G, A X\rangle$, while the extension adds further function symbols and corresponding axioms to the base specification. The idea is to first specify the "pure" objects of the data type and later add operations to be performed on these objects. of course, such an extension should be conservative in the sense that all ground base goals, which are theorems of the extension, are already theorems of the base specification. 
Base Assumption Let $B S I G=\langle S, B O P, P R\rangle$ be a subsignature of $5 I G$ and $B A X$ $\subseteq A X$ be a set of clauses over BSIG. The conditional equations of BAX are denoted by EBAX EBAX is supposed to be symmetric, i.e. for each conditional equation $t \equiv t^{\prime} \Leftarrow \gamma$ in $B A X, t^{\prime} \equiv t \Leftarrow \gamma$ is in $B A X$, too. $\langle B S I G, B A X\rangle$ is called the base specification. Terms, substitutions, atoms and goals over BSIG are called base terms, base substitutions, base atoms resp. base goals. $\langle B S \mid G, B A X\rangle$-theorems are called base theorems. OP-BOP and $A X-B A X$ are denoted by NOP resp. NAX NAX is supposed to consist of conditional equations, and for all $s \in S, G T(B S I G)_{5}$ is assumed to be nonempty.

\section{REDUCTION AND NARROWING}

Two refinements of the Horn clause calculus gain increasing significance in logic programming and theorem proving: reduction (rewriting) of terms and narrowing of equations. In the sequel, reduction is regarded as a bottom-up inference system for the deductive theory, while narrowing is used to derive goal solutions in the deductive theory. Compieteness of reduction is equivalent to a Church-Rosser property of $\langle S \mid G, A X\rangle$. Completeness of narrowing requires a Church-Rosser property. To make this clear let us recall the equational case:

Let $\mathrm{t} \equiv \mathrm{t}^{\prime}$ be an equation to be proved. The reduction approach proceeds by rewriting $t$ and $t$ ' via the equations of $A X$ until both terms have been rewritten into the same term $u$. The Church-Rosser property says that each equational theorem can be proved in this way. We reformulate this procedure by saying that the equation $t \equiv t^{\prime}$ is reduced to the equation $u \equiv u$.

Keeping to this definition of the Church-Rosser property one observes that many specifications are not Church-Rosser. Several variants of the reduction approach have been proposed to cope with this problem (cf. /Padawitz/, chapter 7). Some of them strongly refer to sufficient conditions for the Church-Rosser property (confluence, critical pair convergence,...). Others make use of a modified Church-Rosser definition: Rewriting is confined to non-base equations, and the reduction process terminates as soon as a $\langle S \mid G, B A X\rangle-$ theorem $u=u^{\prime}$ has been obtained. In this case $u$ and $u^{\prime}$ are required to be "normal" terms where "normality" serves as a criterion to stop the reduction process. In some approaches normality coincides with NAX-irreducibility, in other cases normal terms are exactly the base terms. This gives rise to the following two notions of reducedness and reducibility:

\subsection{Goal reducibility}

A goal $\gamma$ is $A X$-reduced if $\gamma$ is a $\langle S I G, B A X$-EBAX)-theorem. (Consequently, in case that BAX consists of conditional equations, i.e. BAX-EBAX is empty, an 
equation $t=t^{\prime}$ is $A x$-reduced if and only if $t=t^{\prime}$.)

Suppose that a set NI of normal terms including all base terms has been separated from the set of all terms. A goal $\gamma$ is NAX-reduced if $\gamma$ is a $\langle S I G, \mathrm{BAX}\rangle$-theorem such that for all $\mathrm{P}\left\langle\mathrm{t}_{1}, \ldots, \mathrm{t}_{n}\right\rangle \in \gamma$ and all $i \leq i \leq n, t_{i}$ is normal.

Neither AX-reducedness implies NAX-reducedness nor NAX-reducedness implies AX-reducedness. Just if EBAX is empty and thus NAX contains all conditional equations of $A X$, then NAX-reducedness is a special case of Ax-reducedness.

Given a goal $\gamma$, a reduction strategy selects a reduction redex of $\gamma$. But it is not the redex structure that matters, it is the redex position. Following the data type LISPPOSITION given by /Broy/, section 6.2: Let $x_{0}$ be a fixed variable, $\delta$ be a goal or term, t be a term and $\gamma=\delta\left[t / x_{0}\right]$. The expression $\delta$ t is called a position over $5 G$ in $\gamma$ if $\delta$ contains $x_{0}$ exactly once. The set of positions over SIG is denoted by Pos(SG).

By the following inference rules, term reduction and goal reducibility are defined simultaneously: Let $A X^{\prime} \in\{A X, N A X\}, S T: G 0 a l(S \mid G) \rightarrow P O S(S I G)$ be a partial function, $\gamma$ be a goal and $t, t^{\prime}$ be terms. $t \rightarrow{ }_{A x^{\prime}} t^{\prime}$ is an $A X^{\prime}$-reduction via ST and $\gamma$ is $A x^{\prime}-r e d u c i b l e v i a S T$ if the expression $t \underset{\mathrm{ACC}}{\mathrm{t}}$ resp. $\frac{S T}{\mathrm{Ax}} \gamma$ is derivable by the following rules:

Term Base Rule $t \underset{\text { RM }}{t}$ for all terms $t$.

Goal Base Rule if $\gamma$ is $A X^{\prime}$-reduced, then $\frac{S T}{A X} \gamma$.

Term Reduction Rule Let $u \equiv u^{i} \Leftarrow g \in A x^{\prime}, t=v\left[u[f] / x_{0}\right], t^{\prime}=v\left[u[f] / x_{0}\right]$ and $\frac{5 T}{A X} g[f]$. Then $t \underset{f x}{\longrightarrow} t$.

Goal Reduction Rule Let $u \equiv u^{\prime} \Leftarrow \vartheta \in A X^{\prime}, S T(\gamma)=\delta * u[f]$ be a position in $\gamma$, i.e. $\gamma=\delta\left[u[f] / x_{0}\right]$. Let $\gamma^{\prime}=\delta\left[u[f] / x_{0}\right] u g[f]$. Then $\frac{S T}{A x^{\prime}} \gamma^{\prime \prime}$ implles $\frac{S T}{A x} \gamma$.

Composition Rule $t_{\overrightarrow{A X}} t^{\prime}$ and $t^{\prime} \overrightarrow{A x} t^{\prime \prime}$ imply $t_{\overrightarrow{A X}} t^{\prime \prime}$.

$y$ is $A X^{\prime}$-reducible if $\gamma$ is $A X^{\prime}$-reducible via some ST. If $u \equiv u^{\prime} \Leftarrow \mathcal{F} \in A X^{\prime}$ and $\vartheta[f]$ is $A X^{\prime}$-reducible, then the positions v*u[f] and $\delta \cdot u[f]$ are called Axi-reduction redices of $v\left[u[f] / x_{0}\right]$ resp. $\delta\left[u[f] / x_{0}\right]$. v[u[f] $\left./ x_{0}\right]$ and all maximal terms of $U[f]$ are called direct $A X^{\prime}-5 T$-successors of $v\left[u[f] / x_{0}\right]$. A goal resp. term is $A X^{\prime}$-irreducible if it has no AX-reduction redex. A substitution $f$ is $A X^{\prime}$-irreducible if for al] $x \in X, f x$ is $A X^{\prime}$-irreducible. The set of all term pairs $\left\langle t, t^{\prime}\right\rangle$ where $t^{\prime}$ is a direct $A X^{\prime}-5 T$-successor of $t$ is called the reductive AX'-ST-ordecing (cf. Jouannaud, Waldmann/).

Soundness of reduction follows immediately, i.e. if $t \frac{S T}{A X^{\prime}} t^{\prime}$ resp. $\frac{S T}{A X^{\prime}} \gamma$, then $t \equiv t^{-1}$ resp. $\gamma$ is a $\langle S I G, A X\rangle$-theorem. Of course, the reducibility of $\gamma$ is equivalent to the existence of term reductions leading from $\gamma$ to a reduced goal, i.e. if $P\left\langle t_{1}, \ldots, t_{n}\right\rangle$ is $A X^{\prime}$-reducible, then there are $A X^{\prime}$-reductions $t_{1} \overrightarrow{A X} U_{1}, \ldots$, $t_{n} \overrightarrow{A X} u_{n}$ such that $P\left\langle u_{1}, \ldots, u_{n}\right\rangle$ is $A X^{\prime}$-reduced.

$A X$ is (ground) $A X$-Church-Bosser if all (ground) (SIG, AX)-theorems are $A X$-reducible. A ground base term t' is a base cepresentation of $t \in T(S I G)$ if the 
equation $t \equiv \mathrm{t}^{\mathrm{t}}$ is a $\langle S \mid G, A X\rangle$-theorem. An atom $\mathrm{P}\left\langle\mathrm{U}_{1}, \ldots, \mathrm{U}_{n}\right\rangle$ is a base representation of $P\left\langle t_{1}, \ldots, t_{n}\right\rangle \in A t(S G)$ if for all $i \leq i \leq n u_{i}$ is a base representation of $t_{i}$. $A$ goal $\gamma$ is base-representable if all atoms of $\gamma$ have a base representation. AX is (ground) NAX-Church-Rosser if all base-representable (ground) $2 S 1 G, A X\rangle-$ theorems are $N A X-r e d u c i b l e$.

The motivation and a number of sufficient conditions for these ChurchRosser properties based on the analysis of "critical pairs" can be found in /Padawitz/, chapter 9 . The following result comes up immediately: If $A X$ is ground $A X^{\prime}$-Church-Rosser and for all $t \equiv t^{\prime} \Leftarrow \delta \in A X^{\prime}$, $t$ is not a base term, then $\langle S I G, A X\rangle$ is a conservative extension of the base specification (cf. section 2.2).

Next we generalize to goal reduction the well-known fact that in the presence of Church-Rosser systems all wellfounded strategies work "the same".

Lemma 3.2.1 Suppose that $A X$ is ground $A X^{\prime}$-Church-Rosser and either (1) $A X^{\prime}=A X$ and for all $t \equiv t^{\prime} \Leftarrow \delta \in A X$ and $q \Leftarrow \lambda \in B A X-E B A X t$ is not a variable and root $(t)$ does not occur in $a$, or

(2) $A X^{\prime}=N A X$, all ground normal terms are $N A X$-irreducible and NT is closed under $B A X$, i.e. for all ground $\langle S G, B A X\rangle$-theorems $t \equiv t^{\prime}$, $t$ is normal iff $t^{\prime}$ is normal.

Let $t \underset{\mathrm{AX}}{\mathrm{t}} \mathrm{t}^{1}$ and $\gamma[\mathrm{t} / \mathrm{x}]$ be a ground goal. If $\gamma[t / x]$ is Ax-reducible, then $\gamma\left[\mathrm{t}^{1} / \mathrm{x}\right]$ is $A X$-reducible, too. If $\gamma[t / x]$ is $N A X$-reducible and has a base representation, then $\gamma\left[t^{\prime} / x\right]$ is $N A X-r e d u c i b l e$, too.

The preceding lemma immediately entails that the order of reduction steps does not matter if $A X^{\prime}$ is Church-Rosser. In other words, each strategy to select reduction redices is complete. Let us call a partial function ST:Goal(SIG) $\rightarrow$ Pos(SIG) an AX'-reduction strategy if for each ground goal $\gamma$, either ST $(\gamma)$ is an AX'-reduction redex of $\gamma$ or ST $(\gamma)$ is undefined. ST is fUll if for each ground goal $\gamma$, ST $(\gamma)$ is defined whenever $\gamma$ has an AX'-reduction redex.

Theorem 3.2 .2 (Reduction strategy independence) Let ST be a full $A X^{\prime}$-reduction strategy and $\gamma$ be a ground goal. Suppose that $A X$ is ground $A X^{\prime}$-Church-Rosser, the reductive $A X^{\prime}-5 T$-ordering is wellfounded on ground goals and either

(1) $A X^{\prime}=A X$ and for all $t \equiv t^{\prime} \Leftarrow \delta$ in $A X$ and $q \Leftarrow \lambda$ in $B A X-E B A X$, $t$ is not a variable and root(t) does not occur in $\mathrm{a}$, or

(2) $A X^{\prime}=N A X$, all ground normal terms are $N A X$-irreducible base terms, NT is closed under $B A X$ and $\gamma$ is base-representable.

Then $\gamma$ is $A X^{\prime}$-reducible via ST if $\gamma$ is $A X^{\prime}$-reducible. 


\subsection{Narrowing solutions}

Narrowing is reduction plus substitution: Prefixes of reduction redices are completed to the full redices by substituting into variables. However, the prefix must contain at least one operation symbol. Otherwise only the substitution would be reduced and the original (non-instantiated) goal would not be affected at all. Moreover, redices could be generated ad infinitum. Completeness of narrowing relies on a Church-Rosser property. According to which is avallable (the $A X$ - or the NAX-Church-Rosser property) there are two ways to derive solutions of $\gamma$ : They are obtained either by substitution and AX-reduction or by substitution and NAX-reduction. Hence the inference rules for narrowing evolve from the rules for reduction (cf. section 3.1 ):

Let ST : Goal(SIG) $\rightarrow$ Pos $(S I G)$ be a partial function, $\gamma, \delta$ be goals and $f$ be a substitution. $\gamma \mid \frac{5 T}{g x^{\prime}}\left\langle\gamma^{\prime}, f\right\rangle$ is an $A X^{\prime}$-narrowing expansion via SI if the expression $\gamma \mid \frac{5 T}{R x^{\prime}}\left\langle\gamma^{\prime}, f\right\rangle$ is derivable by the following rules:

Base Rule if $\gamma[f]$ is $A X^{\prime}$-reduced, then $\gamma \mid \frac{S T}{A X}\langle\phi, f\rangle$.

Narrowing Rule Let $u \equiv u^{\prime} \Leftarrow g \in A X^{\prime}, S T(\gamma)=\delta \cdot t$ be a position in $\gamma$ such that $t$ is not a variable, and $u[g]=t[f]$. Let $\gamma^{\prime}=\delta\left[f\left[u^{\prime}[g] / x_{0}\right]\right] \cup g[g]$. Then $\gamma \mid \frac{S T}{A x^{\prime}}\left\langle\gamma^{\prime}, f\right\rangle$, and $\delta \cdot t$ is an $A x^{\prime}$-narrowingredex of $\gamma$.

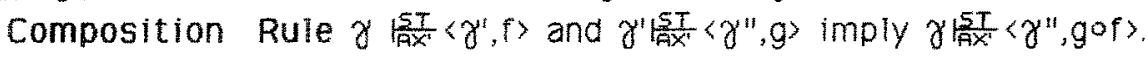

$\gamma \mid \frac{5 T}{A x}\left\langle\gamma^{\prime}, f\right\rangle$ is most general if in all Narrowing Rule applications to derive $\gamma \mid \frac{S T}{A x^{\prime}}\left\langle\gamma^{\prime}, f\right\rangle, f$ is a most general unifier of $\langle t, u\rangle$ (cf. section 2.1). $f$ is an $A X^{\prime}$-narrowing solution viaSI if $\gamma \mid \frac{S T}{A X^{\prime}}\langle\varnothing, f\rangle$.

An $A X^{\prime}$-reduction strategy ST is an AX'-narrowing strategy if for each goal $\gamma$, either ST $(\gamma)$ is an $A X^{\prime}$-narrowing redex of $\gamma$ or ST $(\gamma)$ is undef ined.

Let NS be a set of AX'-irreducible substitutions called normal substitutions such that the identity on $X$ is in NS and for all substitutions $f$ and $g, f \circ g \in N S$ implies $f, g \in N S$. ST is (NS-)uniform if for all goals $\gamma$ and $f \in N$, either ST( $\gamma$ ) and $S T(\gamma[f])$ are defined and $S T(\gamma)[f]=S T(\gamma[f])$ or $S T(\gamma[f])$ is undefined. A detailed discussion of uniformity is given in section 4 below. Uniformity ensures that narrowing is complete with respect to the deductive theory.

Theorem 3.3.1 (Completeness of narrowing with strategy) Let ST be a full and NS-uniform $A X^{\prime}$-narrowing strategy. Suppose that $A X$ is ground $A X^{\prime}$-Church-Rosser, the reductive $A X^{\prime}-S T$-ordering is wellfounded on ground goals and either

(1) $A X^{\prime}=A X$ and for all $t=t^{\prime} \Leftarrow \delta$ in $A X$ and $q \Leftarrow \lambda$ in $B A X-E B A X, t$ is not a variable and root $(t)$ does not occur in $a$, or

(2) $A X^{\prime \prime}=N A X$, all ground normal terms are NAX-irreducible base terms, NT is closed under BAX and instances of normal terms by normal substitutions 
are normal.

- Let $f \in N S$. If $\gamma \operatorname{loT}_{X X}\langle\Omega, f\rangle$, then $\gamma[f]$ is a $\langle S G, A X\rangle$-theorem.

- Let $f$ be a ground normal substitution. If $\gamma[f]$ is a $\langle S I G, A X\rangle$ - theorem and - in case that $A X^{\prime}$ is $N A X$ - $\gamma[f]$ is base-representable, then there is a most general expansion $\gamma \mid \frac{5 T}{A x^{\prime}}\langle\varnothing, g\rangle$ with $f=$ hog for some $n$.

\section{Strategies}

Having laid down the theoretical basis for strategy-controlled reduction and narrowing let us now investigate how common strategy schemata fit into this framework. By Theorem 3.2.2, it is true that - in the presence of ChurchRosser systems - each terminating reduction strategy works equally well, i.e. reduces a given goal $\gamma$ whenever $\gamma$ is reducible at all. However, completeness of narrowing with strategy (Theorem 3.3.1) is guaranteed only if the strategy is uniform. Unfortunately, computation rules like "leftmost-innermost" or "leftmost-outermost" (cf. Manna/, p. $375 \mathrm{ff}$ ) do not lead automatically to uniform narrowing strategies. To illustrate this let ST be a narrowing strategy, $\gamma$ be a goal and $f, g$ be ground normal substitutions such that, say, $S T(\gamma[f])=\delta \cdot t$ and ST $(\gamma[g])=\varphi \cdot u$. Since $f$ and $g$ are irreducible, $t$ and $u$ overlap $\gamma$ in $\gamma[f]$ resp. $\gamma[g]$ properly, i.e. there are positions $\delta^{\prime} \cdot t^{\prime}$ and $\varphi^{\prime} \cdot u^{\prime}$ in $\gamma$ with $\left(\delta^{\prime} \bullet t^{\prime}\right)[f]=\delta \cdot t$ and $\left(\varphi^{\prime} \bullet u^{\prime}\right)[g]=\varphi \cdot u$ (where for all positions $\lambda \cdot v,(\lambda \bullet v)[f]$, the instance of $\lambda \cdot v$ by $f$, is defined as $\lambda\left[f\left[x_{0} / x_{0}\right]\right] \cdot v[f]$, i.e. the fixed variable $x_{0}$ is "left open" when substituting $f$ into $\lambda$.)
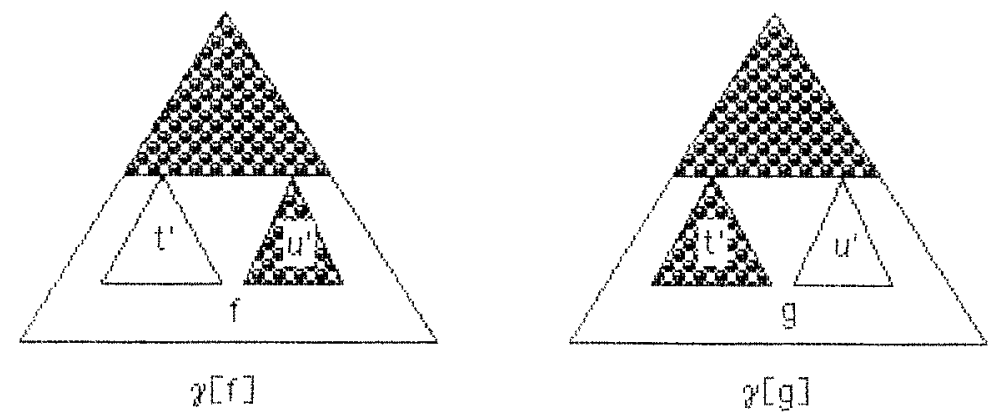

$\gamma$ is given by the polygon consisting of all three inner triangles, while $\delta^{\prime}$ and $\varphi^{\prime}$ are represented by the dotted polygons in the lefthand resp. righthand figure. Provided that ST is uniform, ST $(\gamma)$ is defined,

$$
S T(\gamma)[f]=S T(\gamma[f])=\delta \cdot t=\left(\delta^{\prime} \cdot t^{\prime}\right)[f]
$$

and

$$
S T(y)[g]=S T(\gamma[g])=\varphi \cdot u=\left(\varphi^{\prime} \cdot u^{\prime}\right)[g] .
$$

Since ST $(\gamma), \delta^{\prime} \cdot t^{\prime}$ as well as $\varphi^{\prime} \cdot u^{\prime}$ are positions in the same goal, we obtain $\operatorname{sT}(\gamma)=\delta^{\prime} \cdot t^{\prime}=\varphi^{\prime} \cdot u^{\prime}$. Therefore ST $(\gamma[g])=\left(\delta^{\prime} \cdot t^{\prime}\right)[g]$, and thus $\left(\delta^{\prime} \cdot t^{\prime}\right)[g]$ and $\left(\delta^{\prime} \cdot t^{\prime}\right)[f]$ are reduction redices! since $\gamma, f$ and $g$ were chosen arbitrarily, we 
have obtained a necessary condition for uniformity, namely:

(UNI) For all goals $\gamma$, ground normal substitutions $f, g$ and positions $\lambda \cdot v$ in $\gamma,(\lambda \cdot v)[f]$ is a reduction redex iff $(\lambda \cdot v)[g]$ is a re, iuction redex.

Example 4. Let $\mathrm{NS}=\mathrm{T}(\{0, \mathrm{~s}\})^{\times}$and $\mathrm{A} x^{\prime}$ consist of the equations $\times$ true $\equiv$ true, $c \equiv$ true and $\tau(s(y)) \equiv \tau(y)$. Let $\gamma$ be the equation $\tau(z) \vee c \equiv$ true. Suppose that ST works "leftmost-outermost". Though $0 / z$ is a ground AX'-narrowing solution of $\gamma, O / z$ cannot be computed by ST, Instead, ST will substitute $s(y)$ into $z$ and apply the equation $\tau(s(y)) \equiv \tau(y)$, which leads to a variant of the initial goal, namely to $\tau(y) \sim c \equiv$ true. This follows from non-uniformity of ST: We have

$$
S T(\gamma)=\left(x_{0} \vee c \equiv \text { true }\right) \cdot \tau(z),
$$

but

and thus

$$
\operatorname{sT}(\gamma[0 / z])=\left(\tau(0) \vee x_{0} \equiv \text { true }\right) \cdot c
$$

$$
S T(\gamma)[0 / z]=\left(x_{0} \vee c \equiv \text { true }\right) \cdot \tau(0),
$$

which is different from $\mathrm{ST}(\gamma[0 / z])$. However, $\mathrm{ST}(\gamma)$ corresponds to a reduction redex of another ground instance of $\gamma$, namely to $\gamma[s(0) / z]$ :

$$
\mathrm{sT}(\gamma[\mathrm{s}(0) / z])=\left(x_{0} \vee c \equiv \mathrm{true}\right) \cdot \tau(\mathrm{s}(0)) \text {. }
$$

So we have a position $\lambda \cdot v$ in $\gamma$, namely $\left(x_{0} v C \equiv t r u e\right) \cdot z$, and two ground substitutions $f$ and $g$, namely $s(0) / z$ and $O / z$, such that

is a reduction redex, while

$$
(\lambda \cdot v)[f]=\left(x_{0} v c \equiv \text { true }\right) \cdot \tau(s(0))
$$

$$
(\lambda \cdot v)[g]=\left(x_{o} \vee c \equiv \text { true }\right) \cdot \tau(0)
$$

is not a reduction redex. Hence (UNI) is violated.

The previous example suggests the conjecture that (UN1) is sufficient for uniformity of leftmost-outermost narrowing. Let us now make precise the term "leftmost-outermost".

Given a goal $\gamma$ and a set $M$ of positions in $\gamma, \delta \cdot t \in M$ is leftmost-outermost w.r.t. $M$ if for all $\varphi \cdot u \in M$ the unique occurrence of $x_{0}$ in $\delta$ is lexicographically less than or equal to the unique occurrence of $x_{0}$ in $\varphi$. We conclude from the definition of occurrences in a goal (cf. section 2.1) that "leftmost-outermost" refers to the set of maximal terms in $\delta$, i.e. an occurrence of $x_{0}$ in $\delta$ is leftmost-outermost if $\delta$ contains an atom $P\left\langle t_{1}, \ldots, t_{n}\right\rangle$ in $\delta$ such that for some isisn, all goals $\varphi$ (with unique occurrence of $x_{0}$ ), all atoms $Q\left\langle u_{1}, \ldots, u_{k}\right\rangle \in \varphi$ and all $1 \leq j \leq k$ the occurrence of $x_{0}$ in $t_{i}$ is lexicographically less than or equal to the occurrence of $x_{0}$ in $u_{1}$. A strategy searching for leftmost-outermost redices will thus inspect all terms in a goal in parallel, but each of them in the leftmost-outermost manner. Hence the order of arguments of an atom and the order of atoms in a goal are irrelevant. since reduction redices are terms, 
we would not gain anything by taking these orders into consideration. (That the order of atoms in a goal is irrelevant is well-known from resolution; cf. /Lloyd/, section 9).

We define a partial function LO ("leftmost-outermost") from Goal(SIG) to Pos(SIG) as follows: For all goals $\gamma$, let $M(\gamma)$ be the set of positions $\varphi \cdot u$ in $\gamma$ such that $u$ is not a variable and for some ground normal substitution $f$, $(\varphi \cdot u)[f]$ is a reduction redex. If $M(\gamma)$ is empty, then $L O(\gamma)$ is undefined. Otherwise let $L(\gamma)$ be the leftmost-outermost position w.r.t. M( $\gamma)$.

of course, LO is a full narrowing strategy. Moreover, we obtain

Lemma 4.2 If (UNI) holds true, there are ground normal substitutions and NS is closed under composition, then LO is NS-uniform.

(UNI) can be regarded as a generalization of criterion 4.1(2) in /Fribourg/ for the "well-innermost-reducing" property of rewrite systems. The definition of reduction redices allows us to characterize (UNI) by the following requirement to axiom instances:

(UNI-AX)For all terms $t \notin X$ and ground normal substitutions $f, g$ there is an axiom instance $t[f] \equiv u \Leftarrow \delta$ with reducible premise $\delta$ iff there is an axiom instance $t[g] \equiv v \Leftarrow \varphi$ with reducible premise $\varphi$.

If (UNI-AX) does not hold for some $\mathrm{t}[\mathrm{f}] \equiv \mathrm{U} \Leftarrow \delta$, the specification can be extended by a new constant $\perp$ ("bottom") and the "grounding" equation $t[g] \equiv \perp$.

Example 4.3 (cf. Example 4.1) Let $t=\tau(z), f=s(0) / z$ and $g=0 / z$. Then there is an axiom instance $t[f] \equiv u \Leftarrow \delta$ with reducible (here: empty) premise, namely $\tau(s(0)) \equiv \tau(0)$, but there is no axiom instance $t[g] \equiv v \Leftarrow \varphi$. However, adding $\tau(0) \equiv \perp$ to the axioms makes (UNI-AX) valid. Thus leftmost-outermost narrowing will now compute the solution $0 / z$ of $\gamma$ that we did not achieve before The individual narrowing steps are as follows:

$$
\begin{aligned}
& \tau(z) \cup c \equiv \text { true } \frac{5 T}{A X}\langle\perp \vee c \equiv \text { true, } 0 / z\rangle \text {, }
\end{aligned}
$$

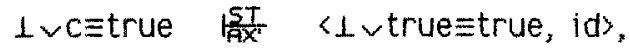

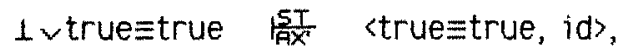

$$
\begin{aligned}
& \text { true } \equiv \text { true } \frac{5 T}{I^{\prime} X^{\prime}}\langle\theta, i d\rangle \text {. }
\end{aligned}
$$

In fact, grounding equations do not violate the soundness of narrowing:

Lemma 4.4 Let t be a ground term, $A X^{\prime \prime}=A X^{\prime} U\{t \equiv 1\}$, NS be the set of $A X^{\prime \prime}-$ irreducible substitutions, ST be an NS-uniform AX"-narrowing strategy, $\gamma$ be a goal and $f \in N S$. In case that $A X^{\prime}$ is NAX, instances of normal terms by normal substitutions are supposed to be normal. Let $f$ be an $A X$ "-narrowing solution of $\gamma$ via ST. Then $\gamma[f]$ is $A X^{\prime}-$ reducible via ST. 
The extension of $A x$ by $t \equiv 1$ preserves the completeness of narrowing provided that the Church-Posser property carries over from $A X^{\prime}$ to $A X^{\prime \prime}=$ $A x \cup\{t \equiv \perp\}$ (ci. Theorem 3.3.1). Consequently, grounding equations cannot be added for free. They should only replace "missing" axiom instances which violate (UNI-Ax). It might be a subject of further research to define axiom schemata where such axiom instances can be detected easily. For instance, /Fribourg/ keeps to axioms of the form $\sigma\left\langle t_{1}, \ldots, t_{n}\right\rangle \equiv t$ where $\sigma\left\langle t_{1}, \ldots, t_{n}\right\rangle$ is "innermost", i.e. $\sigma$ is a non-base operation and $t_{1}, \ldots, t_{n}$ are base terms. In this case - and provided that normal substitutions are base substitutions (UNI-AX) is guaranteed by adding a grounding equation $t \equiv \perp$ for each ground innermost term $t$ which does not match the lef thand side of some axiom.

Closing this section we combine Theorem 3.3.1 and Lemma 4.2 to obtain sufficient conditions for the completeness of leftmost-outermost narrowing:

\section{Theorem 4.5 (Completeness of leftmost-outermost narrowing)} Suppose that $A X$ is ground $A X^{\prime}$-Church-Rosser, the reductive $A X^{\prime}$-LO-ordering is wellfounded on ground goals, (UNI-AX) holds true, there are ground normal substitutions, NS is closed under composition and either

(1) $A X^{\prime}=A X$ and for all $t \equiv t^{\prime} \Leftarrow \delta$ in $A X$ and $q \Leftarrow \lambda$ in $B A X-E B A X t$ is not a variable and root(t) does not occur in $a$, or

(2) $A X^{\prime}=N A X$, all ground normal terms are NAX-irreducible base terms, NT is closed under BAX and instances of normal terms by normal substitutions are normal.

- Let $f \in N S$. If $\gamma \frac{S T}{S x^{\prime}}\langle\not, f\rangle$, then $\gamma[f]$ is a $\langle S G, A X\rangle$-theorem.

- Let $f$ be a ground normal substitution. If $\gamma[f]$ is a $\langle S I G, A X\rangle$-theorem and - in case that $A X^{:}$is NAX - $\gamma[f]$ is a base-representable, then there is a most general expansion $\gamma \mid \frac{S T}{A X^{\circ}}\langle B, g\rangle$ with $f=h \circ g$ for some $h$.

\section{CONCLUSION}

Reduction and narrowing have been generalized from term transformation rules to goal derivation rules. The application of rules is controlled by a strategy given by a partial function $S T:$ Goal(SIG) $\rightarrow$ PoS $(S I G)$ that selects a unique redex in each goal. (Nondeterministic strategies would correspond to a union of such partial functions.) Under the assumption of a Church-Rosser property all terminating reduction strategies work equally well (Theorem 3.2.2). Unfortunately, this result cannot be carried over to narrowing automatically. Although every reduction strategy ST, which selects narrowing redices, yields a narrowing strategy, the completeness of narrowing via ST requires that ST 
is uniform: Given two instances $\gamma[f]$ and $\gamma[g]$ by normal substitutions $f$ resp. $g$ of the same goal $\gamma$, ST must return the same "location" in $\gamma$. In section 4 , we derived (UNI) as a necessary condition for the existence of uniform narrowing strategies. Furthermore, (UNI) was shown to be sufficient for uniformity of leftmost-outermost narrowing. Finally, (UNI) was characterized by (UNI-AX), a property that refers to axiom instances and suggests to replace "missing" axiom instances by "grounding" equations. Further research should be devoted to a complexity analysis of different narrowing strategies in connection with appropriately chosen axiom schemata.

\section{REFERENCES}

/Broy/ M. Broy, On Modularity in Programming, Proc. IFIP 25th Anniversary Celebrations, North-Holland (1985)

/Ehrig, Mahr/ H. Ehrig, B. Mahr, Foundations of Algebraic Specification 1:

Equations and Initial Semantics, Springer (1985)

/Fay/ M. Fay, First Order Unification in an Equational Theory, Proc. 4th Workshop on Automated Deduction, Academic Press (1979) 161-167

/Fribourg/ L. Fribourg, Handling Function Definitions through Innermost Superposition and Rewriting, Proc. RTA '85, Springer LNCS 202 (1985) 325-344 /Huet,oppen/ G. Huet, D. C. Oppen, Equations and Rewrite Rules: A Survey, in: R.

V. Book, ed., Formal Language Theory: Perspectives and Open Problems, Academic Press (1980)

/Hullot/ J. M. Hullot, Canonical Forms and Unification, Proc. 5th CADE, Springer

LNCS 87 (1980) 318-334

/Hußmann/ H. Hußmann, Unification in Conditional-Equational Theories, Proc.

EUROCAL ' 85 , Springer LNCS 204 (1985) 543-553

/Jouannaud, Waldmann/ J.-P. Jouannaud, B. Waldmann, Reductive Conditional

Term Rewriting Systems, Proc. IFIP Formal Description of Programming Concepts 111 (1986)

/Kaplan/ S. Kaplan, Conditional Rewrite Rules, Theoretical Computer Science 33 (1984) 175-194

/Lankford/ D. S. Lankford, Canonical Inference, Report ATP-32, University of Texas at Austin (1975)

Lloyd/J. W. Lloyd, Foundations of Logic Programming (1985)

Manna/ Z. Manna, Mathematical Theory of Computation, MCGraw-Hill (1974)

/Padawitz/ P. Padawitz, Foundations of Specification and Programming with Horn Clauses, to appear

/Slagle/ J. R. Slagle, Automated Theorem-Proving for Theories with Simpli-

fiers, Commutativity and Associativity, Journal ACM 21 (1974) 622-642 